\title{
Portable selfcentred device with calibrated rollers
}

\author{
Adriana Munteanu*, Florentin Cioata, and Emilian Paduraru \\ Technical University Gheorghe Asachi of Iaşi, Department of Machining Tool, Bdul Mangeron, 59A, \\ 700050, Iaşi, România
}

\begin{abstract}
The critical analysis of the self-centering devices for inner cylindrical surfaces highlights a number of advantages and disadvantages of these solutions. The key benefit is the precision but the most important disadvantage it is that doesn't covers a sufficiently range of values for the inner diameters and are very complex. In the present paper a solution of centering mechanism with calibrated rollers that eliminates the disadvantages mentioned above, with a medium complexity but ensuring a sufficient range of values for measured surfaces is proposed. The method used to obtain the specified range of values is graphic method.
\end{abstract}

\section{Introduction}

The inspection of geometrical deviations (especially the coaxial deviation and concentricity deviation) is an issue developed in specified standards $[1,2]$. There are several methods for inspecting but these methods are more or less precise [3].

In industrial practice the measurement of geometrical deviations is an economic aspect that imposes specified inspection methods if the production is mass one. So because of this are used inspection methods more precise that are normally more time consuming and more costly. When the manufacturing production is small the less precise inspection methods that are normally less time consuming and less costly are used [4].

In the coaxiality and concentricity deviation measurement the literature propose in various cases the deviations measuring with the coordinate measuring machines [5] that are time consuming so the development was focused on optical technology, high-speed electronics and computers. But those are expensive. In exclusive production type is necessary a device control that satisfies the following conditions:

- High manoeuvrability, so as to ensure quick measurement of the considered deviation;

- Zeroing quick and easy, without requiring costly additional accessories;

- High degree of universality that would ensure a measuring range as large as possible;

- The indicator instrument: the control device to be fitted with indicator instrument;

- The degree of mobility: control device to be portable;

- Fixing and positioning elements: the control device must have in its structure, fixing and positioning elements for instrument indicator, respectively its orientation relative to the controlled workpiece $[6,7]$.

\footnotetext{
*Corresponding author: adycypmunteanu@yahoo.com
} 


\section{The schematic diagram of the portable device}

In any design process when it is establish a schematic diagram for a control device based on adopted measurement scheme following aspects must be resolved [7, 8, 9]:

- The method of difference application, as a method of measuring of coaxial deviation of inner cylindrical surfaces;

- The compliance of adopted measuring scheme;

- The orientation and the positioning of control device on the inner cylindrical surface specified as datum, in order to materialized its axis;

- To establishing a simple solution for displacement in the radial direction, of the contact elements to the inspected surface;

- The existence, in the device structure, of an element for the realization of inner cylindrical surface tolerated axis;

-The device must be fitted with some indicator whose feeler can perform a circular motion, or if this feeler has a linear movement to establish a variant of displacement transmission of the feeler at the measuring tip;

- To ensure for a sufficiently range values of inner diameters of the two cylindrical surfaces (adjusting solution);

- The possibility to zeroing the control device with calibrated elements accurate mounting on the device;

The proposed solution of selfcentering mechanism (presented in a previous paper [3]) provide greater accuracy of centering and covers a sufficiently typo dimensional values for the cylindrical inner surfaces diameter and is characterized by a high degree of universality (Figure 1).
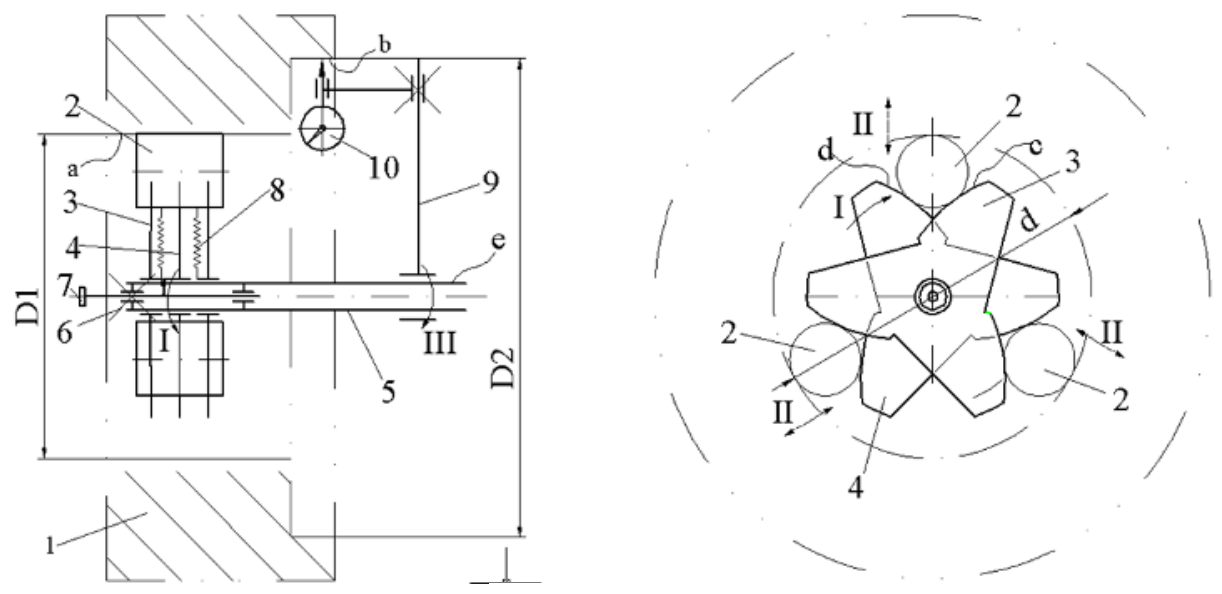

Fig. 1. Portable device

For generation of schematic diagrams of this portable control device, adopted by the measuring scheme, it has been identified a number of requirements to be met (Figure1):

- It is necessary to materialize the axis of inner tolerated cylindrical surface ( $a$ and $b$ surfaces); this requirement is imposed by the adopted scheme measurement;

- It is necessary to materialize the axis of inner cylindrical specified as datum (by the calibrated rollers 2 , and the mobile and fix disks 3 and 4); 
- The measuring of deviation from coaxiality of inner cylindrical surfaces is achieved with a portable control device (with the cylindrical body 5) that must provide both orientation and positioning relative to the workpiece (1) and the measurement;

- Performing measurement of deviation from coaxiality (distance between the materialized rotation axes) using an indicating instrument (the indicating instrument 10 and its support 9);

- Providing guidance of the control device on inner cylindrical surfaces of different diameters (the active surface $e$ );

-Ensure the measuring deviation from coaxiality of inner cylindrical surfaces of parts with different distances between the cylindrical surfaces considered (by movement of calibrated rollers by hand wheels 7).

\section{The graphic solution for control device dimensioning}

Dimensional element of the workpiece that is decisive in determining the characteristic dimensions of the control device is the diameter $D$ of the inner cylindrical surface of the workpiece because it determines the overall dimensions of the control device. To establish the characteristic dimensions of the device control it was considered the nominal values for diameter $D$ of the inner cylindrical surface of the workpiece. This range of values is set between 90 and $130 \mathrm{~mm}$.

The decisive size in determining the overall dimensions of the self centering mechanism with calibrated roller from the control device structure is the minimum diameter of the cylindrical inner surface, in which the centering mechanism should be introduced. Thus, the diameter of this mechanism must have the diameter less than the diameter of the cylindrical inner surface.

The characteristics dimensions of the control device are dimensions that determine the overall of all assemble sizes which determine the structure of the device subassemblies, the lengths of running components who execute displacements, etc. After the obtaining mode, the characteristic dimensions of the device control fall into two distinct categories:

- The characteristic dimensions adopted;

- The characteristics dimensions calculated.

In the considered situation an important place in the control device designed the following adopted characteristic dimensions are relevant:

- The diameter of centering mechanism with calibrated roller (the three fixed and mobile discs, will have a smaller diameter than the minimum diameter of the surface to be inspected;

- The length of the cylindrical body of the self centring device with calibrated rollers (the length of the cylindrical body will be greater than the maximum value of the length of the control);

- The diameter of calibrated rollers. Calibrated rollers, when their generators are brought into contact with the inner cylindrical surface of control, are designed to materialize the cylinder adjacent thereto; by moving their radials to come into contact with the inner cylindrical surface.

But for this selfcentering device the calculated characteristics dimensions depends on the size characteristic of the device control, for ensuring that measurement functions, the orientation of control device for limiting the course of moving parts that perform rotational motion or translation.

To designed control device must considered the following characteristic dimensions:

- Travel length of calibrated rollers - To materialize the adjacent cylinder of the inner cylindrical surface with different values of diameter, the calibrated rollers must move on 
radial direction with the distance $1_{c}$, called the length of stroke; it is calculated by the relationship 1:

$$
l_{c}=\frac{D_{\max }-D_{\min }}{2}
$$

where $D_{\max }$ and $D_{\min }$ are the extreme diameters of the controlled workpiece.

- The angle of rotation of the mobile disc - The mobile disc determine the displacement on radial direction of the calibrated rollers so as to cover the specified value range for the diameter of the inner cylindrical surface with which they come into contact.

In Table 1 are presented the successive position of the calibrated rollers, corresponding to the rotation of the mobile disc with values from $0^{\circ}$ to $40^{\circ} . \beta$ is the angle between the fix disc 2 and mobile disc 3 in his initial position, when $\alpha$ (the rotation angle of mobile disc) have the value zero (Fig. 2,a).

The solution for determining the rotation angle can be obtained by calculation or graphic representation and in the present paper was chosen the graphics solution. Graphical method for obtaining the angle shown in the Table 1 can be seen in Figure 2.

Table 1. Device dimensioning

\begin{tabular}{|c|c|}
\hline $\begin{array}{c}\text { Graphical } \\
\text { representation }\end{array}$ & Calculation \\
\hline Fig. 2 a) & $\begin{array}{l}\alpha \text { - rotation angle of mobile disc } 3 ; \alpha=0^{\circ} ; \\
\beta \text {-angle between the fix disc } 2 \text { and mobile disc } 3 ; \beta=46^{\circ} \text {; } \\
\text { d- diameter of materialized adjacent cylinder } ; d=130 \mathrm{~mm} \text {. }\end{array}$ \\
\hline Fig. 2 b) & $\begin{array}{l}\alpha \text { - rotation angle of mobile disc } 3 ; \alpha=5^{\circ} ; \\
\beta \text {-angle between the fix disc } 2 \text { and mobile disc } 3 ; \beta=46^{\circ} \text {; } \\
\text { d- diameter of materialized adjacent cylinder } ; d=125 \mathrm{~mm} \text {. }\end{array}$ \\
\hline Fig. 2 c) & $\begin{array}{l}\alpha \text { - rotation angle of mobile disc } 3 ; \alpha=10^{\circ} ; \\
\beta \text {-angle between the fix disc } 2 \text { and mobile disc } 3 ; \beta=46^{\circ} ; \\
d \text { - diameter of materialized adjacent cylinder } ; d=118 \mathrm{~mm}\end{array}$ \\
\hline Fig. 2 d) & $\begin{array}{l}\alpha \text { - rotation angle of mobile disc } 3 ; \alpha=15^{\circ} ; \\
\beta \text {-angle between the fix disc } 2 \text { and mobile disc } 3 ; \beta=46^{\circ} \text {; } \\
\text { d- diameter of materialized adjacent cylinder } ; d=111 \mathrm{~mm}\end{array}$ \\
\hline Fig. 2 e) & $\begin{array}{l}\alpha \text { - rotation angle of mobile disc } 3 ; \alpha=20^{\circ} ; \\
\beta \text {-angle between the fix disc } 2 \text { and mobile disc } 3 ; \beta=46^{\circ} \text {; } \\
\text { d- diameter of materialized adjacent cylinder } ; d=106 \mathrm{~mm}\end{array}$ \\
\hline Fig. 2 f) & $\begin{array}{l}\alpha \text { - rotation angle of mobile disc } 3 ; \alpha=25^{\circ} ; \\
\beta \text {-angle between the fix disc } 2 \text { and mobile disc } 3 ; \beta=46^{\circ} \text {; } \\
\text { d- diameter of materialized adjacent cylinder } ; d=99 \mathrm{~mm}\end{array}$ \\
\hline Fig. 2 g) & $\begin{array}{l}\alpha \text { - rotation angle of mobile disc } 3 ; \alpha=30^{\circ} ; \\
\beta \text {-angle between the fix disc } 2 \text { and mobile disc } 3 ; \beta=46^{\circ} ; \\
\text { d- diameter of materialized adjacent cylinder } ; d=96 \mathrm{~mm}\end{array}$ \\
\hline Fig. 2 h) & $\begin{array}{l}\alpha \text { - rotation angle of mobile disc } 3 ; \alpha=35^{\circ} ; \\
\beta \text {-angle between the fix disc } 2 \text { and mobile disc } 3 ; \beta=46^{\circ} \text {; } \\
\text { d- diameter of materialized adjacent cylinder ; } d=93 \mathrm{~mm}\end{array}$ \\
\hline Fig. 2 i) & $\begin{array}{l}\alpha \text { - rotation angle of mobile disc } 3 ; \alpha=40^{\circ} ; \\
\beta \text {-angle between the fix disc } 2 \text { and mobile disc } 3 ; \beta=46^{\circ} \text {; } \\
\text { d- diameter of materialized adjacent cylinder } ; d=90 \mathrm{~mm}\end{array}$ \\
\hline
\end{tabular}




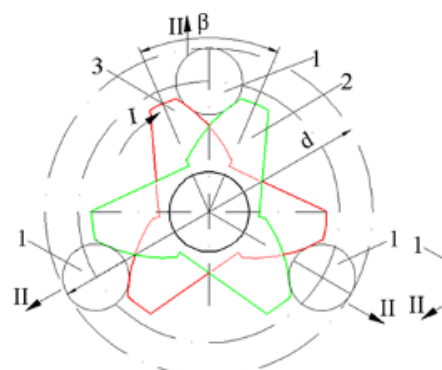

a)

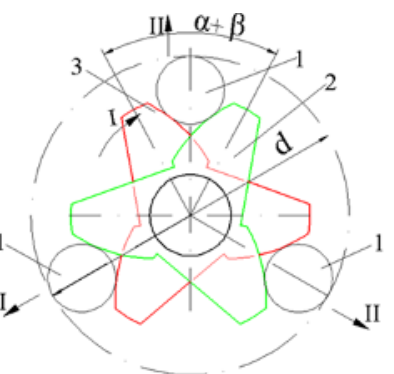

b)

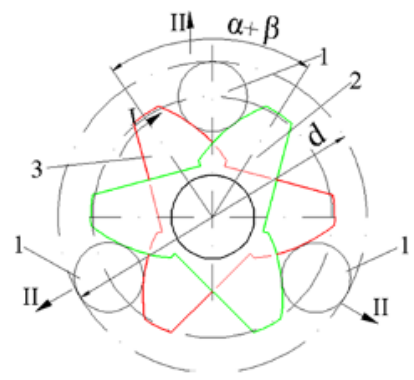

c)

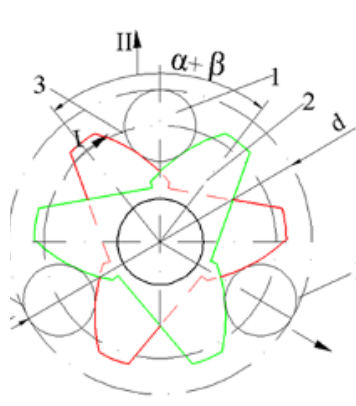

d)

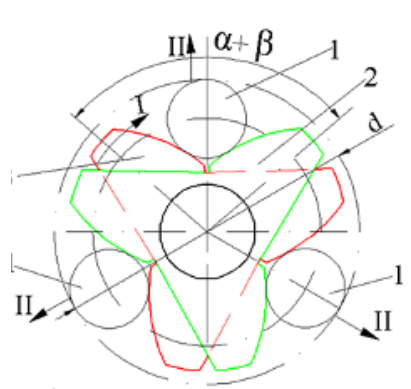

g)

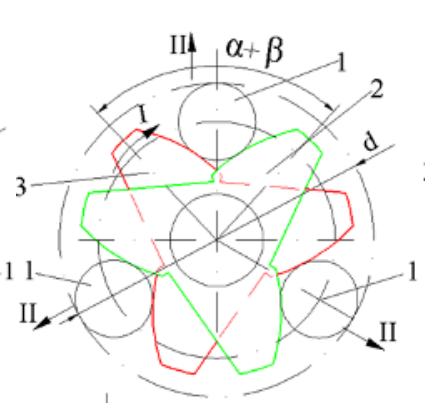

e)

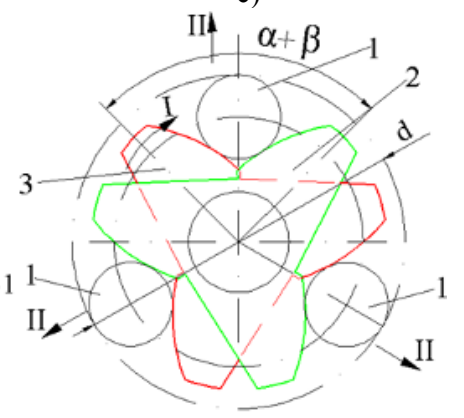

f)

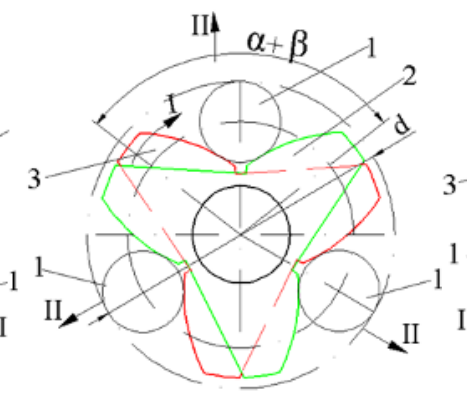

h)

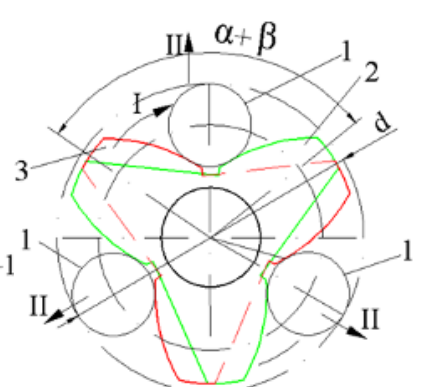

i)

Fig. 2. Graphical dimensioning.

These dimensional characteristics are determined by workpiece dimensions. So must consider:

- Inner diameter cylindrical surface whose axis is specified as a datum axis and determining dimensions orientation- positioning mechanism (subassembly adjacent cylinder to materialize), respectively calibrated roller centering mechanism;

- Inner diameter of the cylindrical surface that measured deviation from concentricity / coaxial and determines the size and positioning mechanism orientation- fixing indicator instrument;

- The values for the total length of the inner cylindrical surfaces of the socket type parts (datum line and tolerated) which determine the length of the cylindrical body of the controller;

- The values for carcass length (distance between walls are charged cylindrical inner surfaces of skeletal parts (baseline and tolerated), which determines the length of the cylindrical body of the controller. 
Considering the fact that the control device is portable, it isn't necessary fixing the workpiece with a mechanism of the control device because it installs (it is oriented and positioned) and fixed on the controlled workpiece, within the area / cylindrical surface / thereof. Therefore, the overall dimensions of the piece to control, respectively, the maximum outer diameter and length of the piece (for parts bushing type) are not decisive in determining the characteristic dimensions of the system.

\section{Conclusion}

In this paper a new approach has been proposed to verify and determine the geometrical specifications. Conceived and designed controller has the following operational and constructive characteristics: the controlled parameter is the form deviation, the measured technique is the direct one, the zeroing is realised on the workpiece, the number of the controlled parameters is one, the device is portable, have the indicating instrument and the mass about half a kilogram.

The focus was on the dimensioning the control device so that to obtain specified values for measured surfaces and the method is the graphic method. In a future work the authors will study and will determine the measurement uncertainties of the control portable device. Crucial issue given that any control equipment is the measurement error which can certify the validity of measurements performed or not, it is essential to determine the error limits with which it will measure. The sources of error will be identified and will be established the specified relationships and the physical model it will be designed. The experimental results regarding the applicability of the developed device will be the purpose of a future work too.

\section{References}

1. ISO 1101:2004 Geometrical product specifications and verification. Geometrical tolerancing. Tolerancing of form, orientation, location and run-out.

2. *** The ISO System of Limits and Fits - Tolerances and Deviations

3. Geometrical Dimensioning and Tolerancing for Design, Manufacturing and Inspection (Second Edition). A Handbook for Geometrical Product Specification using ISO and ASME Standards. Edited by: Georg Henzold, ISBN: 978-0-7506-6738-8

4. Yuhe Li, Yongrong Qiu, Yanxiang Chen, Kaisen Guan. Opt. and Lasers in Eng. 62, 31-37 (2014)

5. X.B. Zhang, X.Q. Jiang, Z. Li.A. Key Eng. Mat., 295-296, 283-288 (2005)

6. Munteanu A., Cioata F. Bul. of Pol. Ins. Iaşi, 62 (2016)

7. T. Damian, Constructive elements for fine mechanics (in Romanian) (Didactic and Pedagogical Publishing House, Bucharest, 1980)

8. N. Seghedin, Technological work devices fine mechanics, (Tehnopress Publishing House, Iasi, 2006)

9. W. Gao, S.W. Kim, H. Bosse, H. Haitjema, Y.L. Chen, X.D. Lu, W. Knapp, A. Weckenmann, W.T. Estler, H. Kunzmann, CIRP Annals - Man.Tech., 64, 2, 773-796 (2015)

10. Y.-P. Cang, G.-J. Wu, A.-M. Xie, W.-J. Wang, Ac. Phot. Sinica 38(11), 2932-2936 (2009) 